\title{
命HAD
}

ISSN-L: 2530-5115

DOI: http://doi.org/10.22585/hospdomic.v2i2.40

\section{Efectos de la inmunoterapia en el paciente adulto en el domicilio: revisión sistemática}

Effects of immunotherapy in the adult patient at home: systematic review

Verónica Domingo Pena', Javier Sanz-Valero',2

1. Universidad Miguel Hernández. Elche. España.

2. Fundación para el Fomento de la Investigación Sanitaria y Biomédica de la Comunidad Valenciana (ISABIALFISABIO). Alicante. España.

Correspondencia/Correspondence

Dr. Javier Sanz-Valero

Departamento de Salud Pública e Historia de

la Ciencia, Universidad Miguel Hernandez,

Campus Sant Joan d'Alacant. Alicante. España.

isanz@umh.es

Recibido/Received

23.01.2018

Aceptado/Accepted

25.02.2018
Conflicto de Intereses/Competing interest La autora y el autor de esta revisión declaran la inexistencia de conflicto de interés

CÓMO CITAR ESTE TRABAJO | HOW TO CITE THIS PAPER

Domingo Pena V. Sanz-Valero J. Efectos de la inmunoterapia en el paciente adulto en el domicilio: revisión sistemática. Hosp Domic. 20 18;2(2):55-65 


\section{RESUMEN}

Objetivo: Revisar la literatura científica relacionada con los efectos de la inmunoterapia en el paciente adulto con atención de salud en el domicilio.

Método: Análisis crítico de los trabajos recuperados mediante revisión sistemática. Los datos se obtuvieron de la consulta directa y acceso, vía Internet, a las siguientes bases de datos bibliográficas del ámbito de las ciencias de la salud: MEDLINE (vía PubMed), The Cochrane Library, Scopus, Cumulative Index to Nursing and Allied Health Literature (CINHAL) y Web of Science. También se hizo uso de la base de datos de la Biblioteca de la Universidad Miguel Hernández. Se consideró adecuado el uso de los Descriptores "Immunotherapy" y "Home Care Services", añadiendo los filtros: "Humans", "Adult 19+ years" y "Comparative Study" o "Clinical Trial". Fecha de la búsqueda: febrero de 2017.

Resultados: Al atribuir los criterios de inclusión y exclusión a la búsqueda se aceptaron 7 estudios para su revisión y análisis crítico. Se evaluó la calidad de los artículos seleccionados mediante el cuestionario CONSORT, obteniendo puntuaciones que oscilaron entre 9 y 18 sobre una puntuación máxima de 25.

Conclusiones: Debido a la falta de artículos coetáneos que abarquen tanto la inmunoterapia como la administración de la misma fuera del servicio hospitalario, así como la presencia de numerosos trabajos que no se pueden enmarcar dentro del grupo de estudios de alta evidencia, mediante este análisis se puede concluir que la terapia actual precisa de nuevos ensayos e investigaciones que permitan encontrar formas más innovadoras de inmunología para poder ser aplicadas a un rango más amplio de patologías inmunitarias, y de esta forma poder generalizarlas a grupos de distintas edades.

Palabras clave: Servicios de atención de salud a domicilio; Servicios de atención a domicilio provisto por hospital; Inmunoterapia; Adulto.

\section{ABSTRACT}

Objective: To review the scientific literature related to the effects of immunotherapy in the adult patient with home health care.

Method: Critical analysis of the works recovered by systematic review. The data were obtained from direct consultation and access, on the Internet, in the following bibliographic databases in the area of health sciences: MEDLINE (via PubMed), The Cochrane Library, Scopus, Cumulative Index to Nursing and Allied Health Literature (CINHAL) and Web of Science. The database of the Miguel Hernández University Library was also used. The use of the Descriptors "Immunotherapy" and "Home Care Services" was considered appropriate, adding the filters: "Humans", "Adult 19+ years" and "Comparative Study" or "Clinical Trial". Date of search: February 2017.

Results: Attributing the inclusion and exclusion criteria to the search, 7 studies were accepted for review and critical analysis. The quality of the selected articles was evaluated through the CONSORT questionnaire, obtaining scores that ranged from 9 to 18 over a maximum score of 25.

Conclusions: Due to the lack of contemporary articles covering both immunotherapy and its administration outside the hospital, as well as the presence of numerous works that cannot be classified within the group of high-evidence studies. This analysis can be concluded that current therapy requires new trials and investigations that allow finding more innovative ways of immunology to be able to be applied to a wider range of immune pathologies, and in this way be able to generalize them to groups of different ages.

Keywords: Home Care Services; Home Care Services, Hospital-Based; Immunotherapy; Adult. 


\section{INTRODUCCIÓN}

El aumento de la cantidad de pacientes, principalmente de edad avanzada, que presentan alguna patología oncológica, ha aumentado considerablemente en los últimos años (1). La inmunoterapia cobra especial importancia en este rango de edad, y los beneficios potenciales de su administración son conocidos por reducir las hospitalizaciones y riesgos posteriores derivados de su no empleo (2).

Sin embargo, tanto la falta de ensayos relativos a la inmunoterapia, como su utilización como terapia domiciliaria o de los beneficios a nivel de calidad de vida (1-3), hace necesaria la actualización de trabajos y de la información obtenida de ensayos previos. Así pues, sería adecuado valorar la presencia o ausencia de dichos ensayos y su pertinencia con respecto al tema que ocupa.

La inmunoterapia sigue siendo una parte esencial de la medicina preventiva y terapéutica, sobre todo en pacientes de edad avanzada. A pesar de esto, la eficacia de la inmunoterapia no ha sido ampliamente investigada y la cantidad de información referente al tema se presume escasa. Numerosos trabajos así lo confirman, como los de Atzpodien et al. (1) y de Gaughran et al. (3), en los cuales se remarca la necesidad de futuros estudios relacionados con el tema dadas las limitaciones que presentaban sus trabajos. Otros estudios (4) solo evalúan los costes y beneficios de un cambio de tratamiento, y muy pocos comentan las ventajas de la terapia administrada en el domicilio de los pacientes.

Ante la ausencia de revisiones sobre el tema, en este trabajo se pretende comprobar los estudios concernientes al tratamiento inmunoterápico y a su aplicación mediante terapéutica a domicilio, así como discernir sobre los posibles resultados derivados de esta búsqueda. En consecuencia, el objetivo de este estudio fue revisar la literatura científica relacionada con los efectos de la inmunoterapia en el paciente adulto que recibe servicios de atención de salud a domicilio.

\section{MÉTODOS}

\section{Diseño}

Estudio descriptivo transversal y análisis crítico de los trabajos recuperados mediante revisión sistemática.

\section{Fuente de obtención de los datos}

Los datos se obtuvieron de la consulta directa y acceso, vía Internet, a las siguientes bases de datos bibliográficas del ámbito de las ciencias de la salud: MEDLINE (vía PubMed), The Cochrane Library, Scopus, Cumulative Index to Nursing and Allied Health Literature (CINHAL) y Web of Science. También se hizo uso de la base de datos de la Biblioteca de la Universidad Miguel Hernández en el campus de Sant Joan d'Alacant.

\section{Tratamiento de la información}

Para definir los términos de la búsqueda se consultó el Thesaurus desarrollado por la U.S. National Library of Medicine. 
Se consideró adecuado el uso de los Descriptores "Immunotherapy" y "Home Care Services". La ecuación de búsqueda final se desarrolló para su empleo en la base de datos MEDLINE, vía PubMed, utilizando los filtros: "Humans", "Adult 19+ years" y "Comparative Study" o "Clinical Trial".

La ecuación de búsqueda final fue:

("Immunotherapy"[MeSH] OR "Immunotherapy"[Title/Abstract] OR "Immunotherapies"[Title/Abstract]) AND ("Home Care Services"[MeSH] OR "Home Care"[Title/Abstract] OR "Domiciliary Care"[Title/Abstract] OR "Home Care Services, Hospital-Based"[Mesh] OR "Hospital-Based Home Care"[All Fields] OR "Hospital Based Home Care"[Title/Abstract] OR "Hospital Home Care Services"[Title/Abstract] OR "Hospital-Based Home Care Services"[Title/Abstract] OR "Hospital Based Home Care Services"[Title/ Abstract] OR "Home Hospitalization"[Title/Abstract] OR "Hospital at Home"[Title/Abstract] OR "Hospital-at-Home"[Title/Abstract] OR "Hospital Home Care"[Title/Abstract] OR "Hospital at Home Care"[Title/Abstract] OR "Hospital in the Home"[Title/Abstract])

Esta estrategia se adaptó a cada una del resto de bases de datos consultadas. La búsqueda se realizó en febrero de 2017 y se completó con el análisis del listado bibliográfico de los artículos que fueron seleccionados.

\section{Selección final de los artículos}

Se escogieron para su estudio los artículos, en cualquier idioma, que cumplieron los siguientes criterios: adecuarse a los objetivos de la búsqueda (inmunoterapia en el domicilio), estar publicados en revistas revisadas por pares y poder recuperar el texto completo del trabajo. Se excluyeron aquellos no realizados en humanos, los que incluían menores de edad o que no tratasen la inmunoterapia como tema principal del estudio.

\section{Evaluación de la calidad metodológica}

Para valorar la calidad de los documentos seleccionados se utilizaron las directrices para la publicación de estudios observacionales CONSORT (CONsolidated Standards Of Reporting Trials) (5), conformado por un listado de 25 aspectos esenciales (ítems) que deben describirse en la publicación de dichos estudios. Para cada artículo se asignó un punto por cada ítem presente (en caso de no ser aplicable no puntuaba). Cuando un ítem estaba compuesto por varios puntos, estos se evaluaban de forma independiente, dándole el mismo valor a cada uno de ellos y realizándoles posteriormente un promedio de tal forma que en ningún caso se pudiera superar la puntuación de un punto por ítem.

\section{Extracción de los datos}

Los autores evaluaron la adecuación de los estudios de forma independiente. Para considerar válido el proceso de selección, se estableció que la evaluación de la concordancia entre ambos autores (índice Kappa) debe ser mayor a 0,60 (buena o muy buena fuerza de concordancia). Siempre que se cumpliera esta condición, cualquier discrepancia se resolvió por consenso entre los autores.

Se usaron dobles tablas para tener control de los datos extraídos; esto permitió la detección de errores y su corrección consultando nuevamente los documentos originales.

Los trabajos se estructuraron según las variables del estudio para sistematizar y facilitar la comprensión de los resultados; se consideraron los siguientes datos: primer autor y año de publicación, 
diseño del estudio, población objetivo, tipo de inmunoterapia, período de seguimiento, país donde se realizó el estudio y principales resultados obtenidos.

\section{RESULTADOS}

De la búsqueda realizada en las diferentes bases de datos, se obtuvieron 123 referencias, de las cuales $1(0,81 \%)$ fue encontrada por búsqueda manual y el resto provenían de las bases de datos consultadas: 11 de MEDLINE (8,94\%), 8 de la Cochrane Library (6,50\%), 2 de la Web of Science (1,63\%), 3 de la base CINAHL (2,44\%) y 98 de Scopus (79,67\%). De todos ellos, 17 artículos $(13,82 \%)$ fueron eliminados por estar repetidos en una o varias bases de datos. Posteriormente, y aplicando los criterios de inclusión y exclusión (figura 1), quedaron 7 trabajos (1-3,6-9) (5,69\%) que cumplieron los requisitos propuestos y por tanto fueron aceptados para su revisión (tabla 1).

Figura 1 - Identificación y selección de estudios
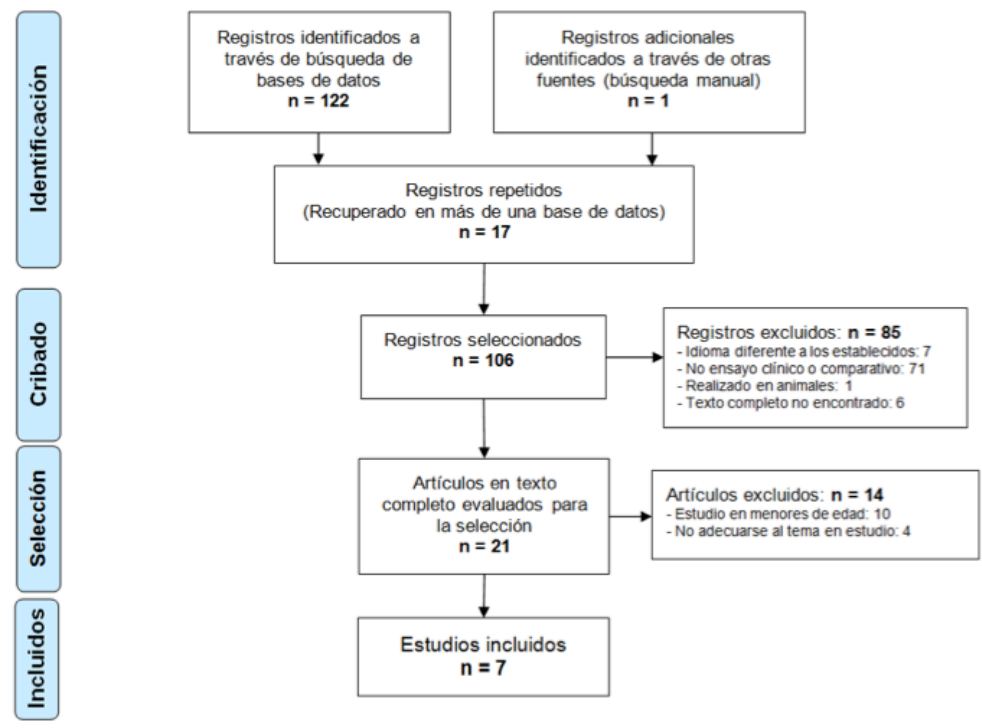

El acuerdo sobre la pertinencia de los estudios seleccionados entre los dos evaluadores fue del 100\%. Se evaluó la calidad de los artículos seleccionados empleando el cuestionario CONSORT, resultando en puntuaciones que oscilaron entre 9,00 y 18,00 (tabla 2).

Los 7 estudios revisados cumplían con el criterio de ser ensayos clínicos, a excepción de dos de ellos que eran comparativos, diseño también aceptado, y provenían de Alemania, Inglaterra y EE.UU., estando todos ellos redactados en inglés. Cuatro de ellos (1,6-8) pertenecían al mismo autor (Atzpodien et al.) realizados entre los años 1991 y 2005. El más reciente de los 7 fue publicado en el año 2007 (3) lo que puede recalcar la falta de estudios que tratan este tema.

La edad media de la población recogida en los trabajos seleccionados superaba los 55 años. 
El periodo de seguimiento osciló entre 3 semanas y 10 años, aunque en los trabajos de Arthur et al. (2) y Gaughran et al. (3) no se tuvo constancia del tiempo.

En estos artículos se comparan tratamientos inmunoterápicos (Interleucina-2 (IL-2) y vacuna contra el virus influenza, principalmente) aplicados en el hogar, a nivel residencial (2) y a nivel ambulatorio (7). En uno de ellos se evaluaba la calidad de vida de los pacientes tras recibir su tratamiento, mediante el Cuestionario de Calidad de Vida de la Organización Europea para la Investigación y Tratamiento del Cáncer (EORTC-QLQ-C30) (6).

El tipo de enfermedad más estudiada fue el carcinoma de células renales $(1,6-8)$ y la gripe, causada por el virus influenza (2,3), y en uno (9) los procesos alérgicos.

Los resultados de la revisión mostraron que la combinación de cuidados a domicilio y vacuna mejora el tratamiento (2). Además la inmunoterapia domiciliaria es más segura y produce menos reacciones adversas graves (9). Se observó una reducción drástica de la toxicidad, además de confirmar la eficacia de la combinación terapéutica subcutánea a bajas dosis (8) e incluso la remisión del tumor a largo plazo (7).

Por otro lado se comprobó que el tratamiento es igualmente efectivo independientemente de la edad del paciente y de la reducción de la dosis de IL-2 que se les aplica (1).

Ahora bien, los beneficios de la readministración de la vacuna del virus influenza permanecen inciertos (3).

Por último, en los 4 trabajos de Atzpodien et al. $(1,6-8)$ se concluía que el tratamiento inmunoterápico administrado de forma ambulatoria puede mejorar la calidad de vida de los pacientes al disminuir la toxicidad sistémica. Este hecho se contrarresta con un artículo posterior, que concluye que la reducción de la calidad de vida en los pacientes referente a su funcionamiento físico, psicología y relaciones sociales tras el tratamiento inmunoterápico ambulatorio puede servir de indicador temprano para la respuesta e incluso para la supervivencia de los pacientes.

\section{DISCUSIÓN}

A pesar de la importancia del tema que se está tratando, se encontraron pocos artículos relacionados que presentasen las características propicias para ser analizados, dado que un gran número de los artículos fueron descartados por no tratarse de estudios de alta evidencia (no eran ensayos clínicos o estudios comparativos) o por no cumplir con las características de edad de los pacientes.

La mayoría de los artículos excluidos pertenecían a la base de datos Scopus, la cual no dispone de Thesaurus, por lo que no permite el uso de Descriptores para la búsqueda precisa de los ensayos, aumentando la aparición de "ruido" en los documentos recogidos tras la pesquisa.

Se destaca la ausencia de revisiones sistemáticas anteriores sobre el tema en cuestión, a excepción de un trabajo de van Haastregt (10) donde se comentó que por las limitaciones del estudio no se pudo concluir de forma certera que hubiera un cambio significativo en la prevención de la enfermedad gracias a las visitas a domicilio. Del mismo modo, otros trabajos como el de Lee et al. (11) o el de Jolles et al. (12) alertaban de la necesidad de ensayos comparativos con diferentes estrategias inmunológicas.

Cabría esperar que las puntuaciones obtenidas en la tabla CONSORT fueran en aumento conforme más recientes fueran las publicaciones, sin embargo se hallaron artículos con mayor antigüedad que presentan mejor puntuación que otros de años posteriores. Esto puede ser debido a la poca exigencia que se requería para la difusión de artículos científicos antes del año 2010, año en el que se publicó la declaración CONSORT (5). 
Los países citados en los artículos fueron Inglaterra, EE.UU. y Alemania, siendo el idioma de las publicaciones el inglés, aunque también se aceptaban artículos en español y portugués. Era de esperar que se encontrasen trabajos principalmente en lengua anglosajona, este idioma es el elegido para la publicación de la mayoría de los artículos ya que hacerlo en otra lengua distinta resulta negativo para la visibilidad, el factor de impacto y las citaciones (13).

La obsolescencia de los trabajos denotó la necesidad de artículos más actualizados, dado que el más reciente se remonta a 2007, y siendo este un tema de gran importancia se esperaban artículos con menor edad. Reseñar que esta obsolescencia es mayor a lo observado en otros campos de las ciencias de la salud (14).

La media de los participantes en los ensayos superaba los 55 años, con lo que se puede deducir que efectivamente este tipo de enfermedades y su tratamiento son más estudiados, o presentan mayor relevancia, en personas cercanas a la tercera edad, ya sea por la sensibilidad de este grupo de edad a las afecciones tratadas en estas publicaciones, por la prevalencia que presentan o la ausencia de ello en otros grupos de edad.

Los tratamientos más nombrados en estos trabajos son IL-2 por vía subcutánea e interferón alpha-2a (IFN-alpha2a) por vía subcutánea para tratar el carcinoma de células renales, además de la vacuna contra el virus influenza. Solo en un trabajo se hablaba de las terapias alérgicas (9), hecho que difiere con la elevada importancia que nombraba Canonica et al. y Bachert et al. sobre el elevado número de personas en Europa con alergia estacional $(15,16)$. Del mismo modo, en uno de los artículos estudiados se ponía de manifiesto la calidad de vida de los pacientes, siendo este el único en donde se cuantificaba el bienestar de los pacientes ante el empleo del tratamiento de elección (6).

Las enfermedades más relevantes en estos estudios fueron la gripe, causada por el virus influenza, y el carcinoma de células renales, siendo recurrente esta última en los trabajos de Atzpodien (6-8), especialista del departamento de oncología.

Se pudo comprobar que en varios artículos rechazados para esta revisión se contemplaban nuevos métodos para la terapia inmunológica, tanto a nivel hospitalario como a nivel domiciliario. En ellos, se evaluaban los beneficios y costes de estas nuevas terapias y de los posibles gastos para el sistema sanitario, como en el caso de los trabajos de M. Haeney (17), Canonica (16) y Bachert (15) en los que se concluyeron que estas terapias eran rentables y que podrían representar una alternativa al tratamiento convencional dado que se brindaba un ahorro en los costos de hospitalización, al no ser necesaria su ocupación.

Los trabajos evaluados pretendían demostrar la eficacia y las mejoras que conlleva la administración de la inmunoterapia a domicilio o de forma ambulatoria, tanto a nivel de remisión de la enfermedad como de reducción de futuras reacciones adversas derivadas e incluso como un marcador de la evolución del paciente. Sin embargo, también se encontraron trabajos que no confirmaban los beneficios ni perjuicios de la terapia propuesta; tal es el ejemplo de la administración de forma repetida de la vacuna del virus influenza en el trabajo de Gaughran et al (3) o los beneficios de la visita de personal de enfermería como propone Dalby et al (18).

A pesar de todo lo expuesto anteriormente, debido a la falta de artículos coetáneos que abarquen tanto la inmunoterapia como la administración de la misma fuera del servicio hospitalario, así como la presencia de numerosos trabajos que no se pueden enmarcar dentro del grupo de estudios de alta evidencia, mediante este análisis se puede concluir que la terapia actual precisa de nuevos ensayos e investigaciones que permitan encontrar formas más innovadoras de inmunología para poder ser aplicadas a un rango más amplio de patologías inmunitarias, y de esta forma poder generalizarlas a grupos mayores de distintas edades. 


\section{BIBLIOGRAFÍA}

1. Atzpodien J, Wandert T, Reitz M. Age does not impair the efficacy of immunochemotherapy in patients with metastatic renal carcinoma. Crit Rev Oncol Hematol. 2005;55(3):193-9. DOI: 10.1016/j.critrevonc.2005.03.001; PMID: 16115777

2. Arthur AJ, Matthews RJ, Jagger C, Clarke M, Hipkin A, Bennison DP. Improving uptake of influenza vaccination among older people: a randomised controlled trial. Br J Gen Pract. 2002;52(482):717-22. PMID: 12236274

3. Gaughran F, Walwyn R, Lambkin-Williams R, Whelan P, Chatterton K, Oxford J, et al. Flu: effect of vaccine in elderly care home residents: a randomized trial. J Am Geriatr Soc. 2007;55(12):191220. DOI: 10.1111/j.1532-5415.2007.01471.x; PMID: 18081669

4. Gardulf A, Andersen V, Björkander J, Ericson D, Frøland SS, Gustafson R, et al. Subcutaneous immunoglobulin replacement in patients with primary antibody deficiencies: safety and costs. Lancet. 1995;345(8946):365-9. PMID: 7845120

5. Eldridge SM, Chan CL, Campbell MJ, Bond CM, Hopewell S, Thabane L, et al. CONSORT 2010 statement: extension to randomised pilot and feasibility trials. BMJ. 2016;355:15239. DOI: 10.1136/bmj.i5239; PMID: 27777223

6. Atzpodien J, Küchler T, Wandert T, Reitz M. Rapid deterioration in quality of life during interleukin-2- and alpha-interferon-based home therapy of renal cell carcinoma is associated with a good outcome. Br J Cancer. 2003;89(1):50-4. DOI: 10.1038/sj.bjc.6600996; PMID: 12838299

7. Atzpodien J, Poliwoda H, Kirchner H. Alpha-Interferon and interleukin-2 in renal cell carcinoma: studies in nonhospitalized patients. Semin Oncol. 1991;18(5 Suppl 7):108-12. PMID: 1948123

8. Atzpodien J, Lopez Hänninen E, Kirchner H, Bodenstein H, Pfreundschuh M, Rebmann U, et al. Multiinstitutional home-therapy trial of recombinant human interleukin-2 and interferon alfa-2 in progressive metastatic renal cell carcinoma. J Clin Oncol. 1995;13(2):497-501. DOI: 10.1200/ JCO.1995.13.2.497; PMID: 7844611

9. Hurst DS, Gordon BR, Fornadley JA, Hunsaker DH. Safety of home-based and office allergy immunotherapy: A multicenter prospective study. Otolaryngol Head Neck Surg. 1999;121(5):55361. 9 DOI: 10.1016/S0194-5998(99)70055-0; PMID: 1054746

10. van Haastregt JC, Diederiks JP, van Rossum E, de Witte LP, Crebolder HF. Effects of preventive home visits to elderly people living in the community: systematic review. BMJ. 2000;320(7237):754-8. DOI: 10.1136/bmj.320.7237.754; PMID: 1072036

11. Lee S, Nolte H, Benninger MS. Clinical considerations in the use of sublingual immunotherapy for allergic rhinitis. Am J Rhinol Allergy. 2015;29(2):106-14. DOI: 10.2500/ajra.2015.29.4148; PMID: 25785750

12. Jolles S, Hughes J, Whittaker S. Dermatological uses of high-dose intravenous immunoglobulin. Arch Dermatol. 1998;134(1):80-6. DOI: 10.1001/archderm.134.1.80; PMID: 9449914

13. Franco-López A, Sanz-Valero J, Culebras JM. Publicar en castellano, o en cualquier otro idioma que no sea inglés, negativo para el factor de. J Negat No Posit Results. 2016;(2):65-70. DOI: 10.19230/jonnpr.2016.1.2.1005

14. Sanz-Valero J, Wanden-Berghe C. Análisis bibliométrico de la producción científica, indizada en MEDLINE, sobre los servicios de salud proporcionados por las unidades de hospitalización a domicilio. Hosp Domic. 1(1);2017:21-34. DOI: 10.22585/hospdomic.v1i1.3 
15. Bachert C, Vestenbaek U, Christensen J, Griffiths UK, Poulsen PB. Cost-effectiveness of grass allergen tablet (GRAZAX) for the prevention of seasonal grass pollen induced rhinoconjunctivitis - a Northern European perspective. Clin Exp Allergy. 2007;37(5):772-9. DOI: 10.1111/j.13652222.2007.02706.x; PMID: 17456225

16. Canonica GW, Poulsen PB, Vestenbaek U. Cost-effectiveness of GRAZAX for prevention of grass pollen induced rhinoconjunctivitis in Southern Europe. Respir Med. 2007;101(9):1885-94. DOI: 10.1016/j.rmed.2007.05.003; PMID: 17611095

17. Haeney M. Intravenous immune globulin in primary immunodeficiency. Clin Exp Immunol. 1994;97(Suppl 1):11-5. PMID: 8033427

18. Dalby DM, Sellors JW, Fraser FD, Fraser C, van Ineveld C, Howard M. Effect of preventive home visits by a nurse on the outcomes of frail elderly people in the community: a randomized controlled trial. CMAJ Can Med Assoc J. 2000;162(4):497-500. PMID: 10701382 


\begin{tabular}{|c|c|c|c|c|c|c|c|c|c|}
\hline \multirow{7}{*}{ 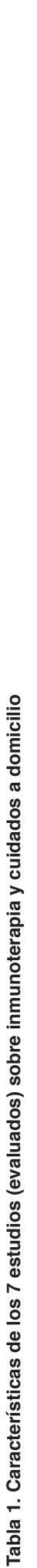 } & 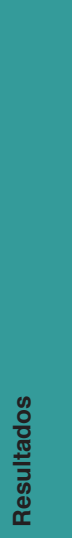 & 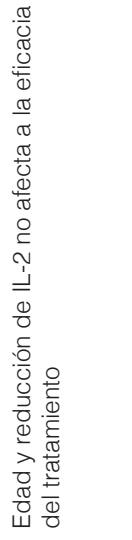 & 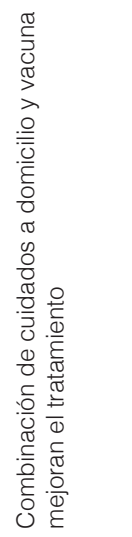 & 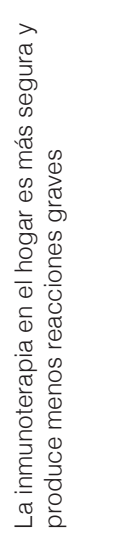 & 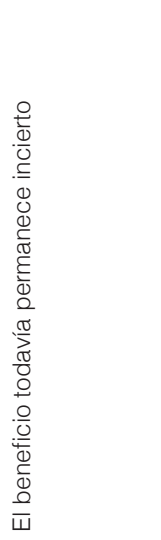 & 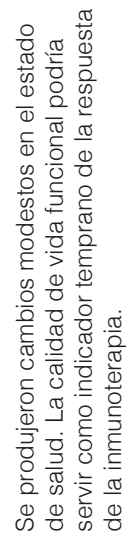 & 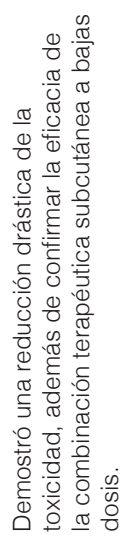 & 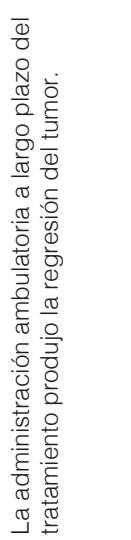 & \multirow{7}{*}{ 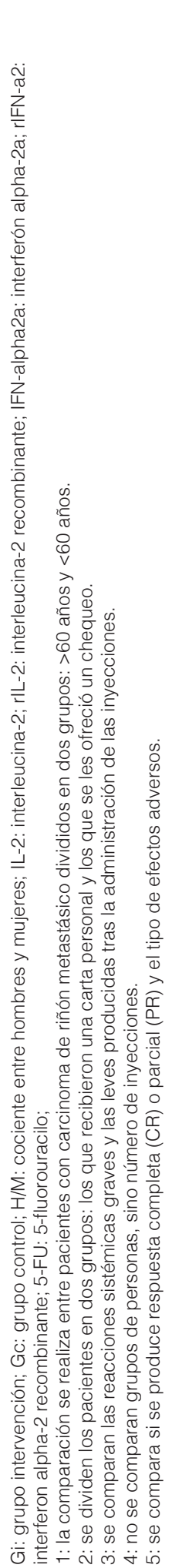 } \\
\hline & $\frac{0,0}{\sigma]}$ & 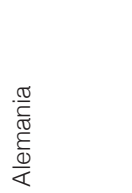 & 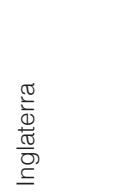 & $\begin{array}{l}0 \\
\frac{0}{0} \\
\frac{0}{5} \\
5 \\
0 \\
\frac{0}{0} \\
\frac{\pi}{0} \\
\frac{\omega}{4}\end{array}$ & $\begin{array}{l}\frac{\pi}{2} \\
\frac{\pi}{0} \\
\frac{\pi}{0} \\
\underline{\Xi}\end{array}$ & $\begin{array}{l}\frac{\pi}{\omega} \\
\tilde{\omega} \\
0 \\
0 \\
2\end{array}$ & 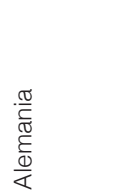 & $\begin{array}{l}\frac{\pi}{0} \\
\tilde{\omega} \\
0 \\
0 \\
0 \\
2\end{array}$ & \\
\hline & 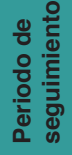 & 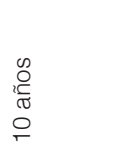 & $\begin{array}{l}\frac{\pi}{0} \\
0 \\
0 \\
0 \\
0 \\
z\end{array}$ & 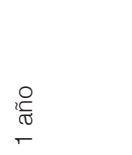 & $\begin{array}{l}\frac{\pi}{0} \\
\text { क } \\
0 \\
0 \\
0 \\
2\end{array}$ & 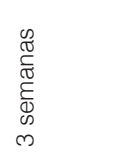 & & 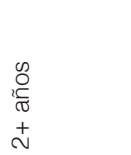 & \\
\hline & 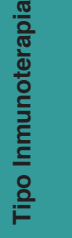 & 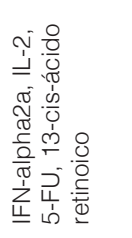 & 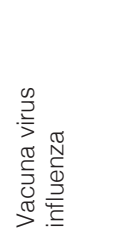 & 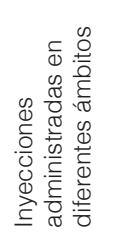 & 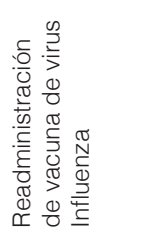 & 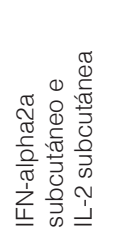 & 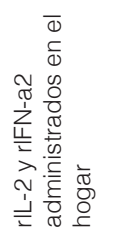 & 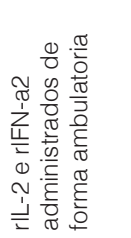 & \\
\hline & $\begin{array}{l}\frac{0}{0} \\
\frac{\pi}{0} \\
\frac{\pi}{0}\end{array}$ & 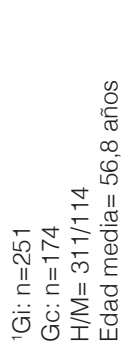 & 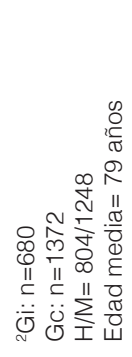 & 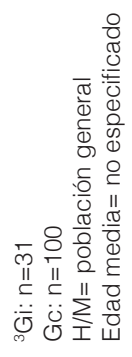 & 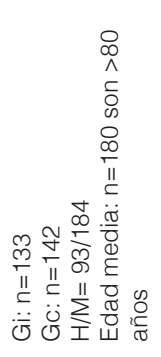 & 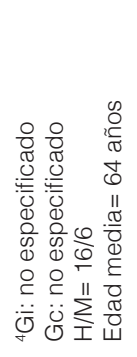 & 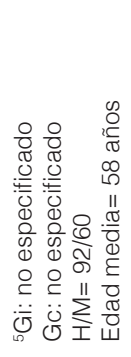 & 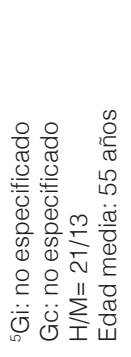 & \\
\hline & 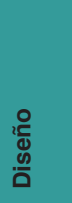 & 总 & 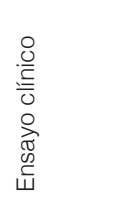 & 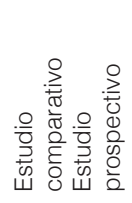 & 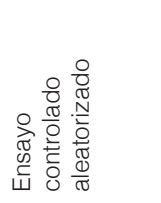 & 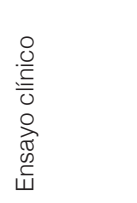 & 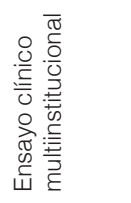 & 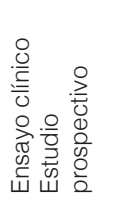 & \\
\hline & 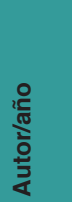 & 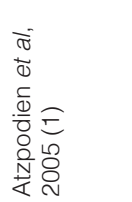 & 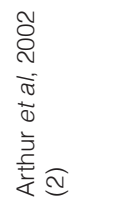 & 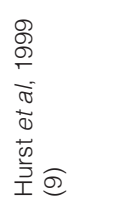 & 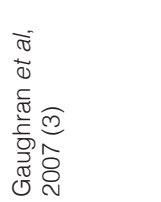 & 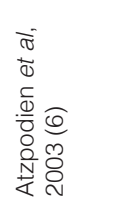 & 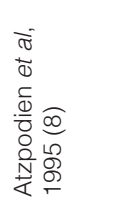 & 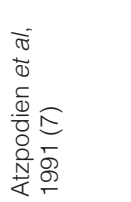 & \\
\hline
\end{tabular}


Efectos de la inmunoterapia en el paciente adulto en el domicilio: revisión sistemática

\begin{tabular}{|c|c|c|c|c|c|c|c|c|}
\hline \multirow{28}{*}{ 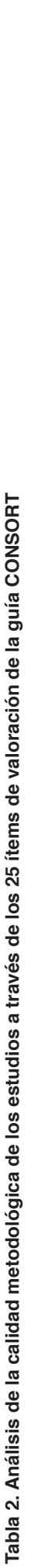 } & ธั॰ & $\overline{6}$ & 8 & $\stackrel{\infty}{\infty}$ & $\cong$ & g & $\widetilde{N}$ & $\stackrel{ल}{\oplus}$ \\
\hline & $\begin{array}{l}\bar{\Xi} \\
\text { 。्̊ }\end{array}$ & $\stackrel{\circ}{\stackrel{ }{\Gamma}}$ & $\begin{array}{l}\circ \\
\stackrel{\circ}{\circ}\end{array}$ & $\stackrel{\text { ?ִ }}{\rightleftharpoons}$ & $\begin{array}{l}\stackrel{\circ}{\infty} \\
\stackrel{\infty}{\leftarrow}\end{array}$ & $\begin{array}{l}\circ \\
\stackrel{\circ}{\circ}\end{array}$ & $\stackrel{\circ}{\stackrel{\leftrightarrow}{\sim}}$ & $\stackrel{\circ}{\circ}$ \\
\hline & นึ & 0 & - & 0 & - & - & 0 & 0 \\
\hline & ন & 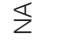 & 0 & $\mathbb{Z}$ & $\circ$ & 0 & 0 & 0 \\
\hline & $\widetilde{N}$ & \& & 0 & $\bar{z}$ & 0 & 0 & 0 & 0 \\
\hline & สี & - & - & - & - & - & 0 & - \\
\hline & $\overline{\mathbf{N}}$ & - & - & - & - & - & - & - \\
\hline & ลิ & - & - & - & - & - & - & 0 \\
\hline & $\stackrel{\circ}{-}$ & 0 & - & - & 0 & 0 & - & 0 \\
\hline & $\stackrel{\infty}{\leftarrow}$ & - & 0 & 0 & - & 0 & - & 0 \\
\hline & 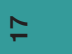 & : & - & 0 & : & - & - & ?ִ \\
\hline & $\stackrel{\varphi}{\circ}$ & - & - & - & - & - & - & - \\
\hline & $\stackrel{20}{\leftarrow}$ & - & - & 0 & $\tau$ & 0 & 0 & $\tau$ \\
\hline & $\stackrel{\nabla}{ \pm}$ & : & 0 & مُ & 0 & 0 & مُ & 0 \\
\hline & $\stackrel{m}{\longrightarrow}$ & - & - & - & - & $\stackrel{2}{0}$ & مُ & مُ \\
\hline & $\cong$ & $\Sigma$ & $\begin{array}{l}L ? \\
0\end{array}$ & $\Sigma$ & - & ?ִ & $\stackrel{10}{0}$ & $\stackrel{\leftrightarrow}{\circ}$ \\
\hline & $=$ & 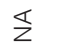 & 0 & 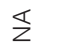 & - & 0 & 0 & 0 \\
\hline & 으 & $\underline{z}$ & 0 & $\underline{z}$ & 0 & 0 & 0 & 0 \\
\hline & $\sigma$ & $\Sigma$ & 0 & $\Sigma$ & 0 & 0 & 0 & 0 \\
\hline & $\infty$ & 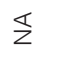 & - & $\Sigma$ & مُ & ○ & 0 & 0 \\
\hline & $\sim$ & 0 & : & $\Sigma$ & - & 0 & 0 & 0 \\
\hline & 0 & 0 & 0 & - & - & : & - & - \\
\hline & n & - & $\tau$ & - & - & $\tau$ & $\tau$ & $\tau$ \\
\hline & $\sigma$ & مُ & مْ & ?ִ & - & $\stackrel{\text { L }}{0}$ & - & ?ִ \\
\hline & $m$ & 0 & مُ & - & - & 0 & $\stackrel{2}{0}$ & 0 \\
\hline & $N$ & - & $\tau$ & - & - & - & $\tau$ & - \\
\hline & $\tau$ & ?ִ & - & : & - & 0 & - & 0 \\
\hline & & 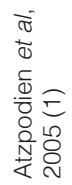 & 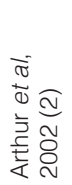 & 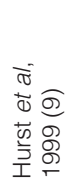 & 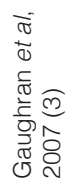 & 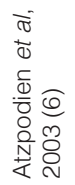 & 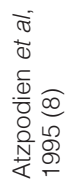 & 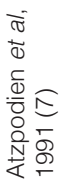 \\
\hline
\end{tabular}

